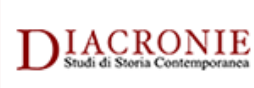

\title{
Diacronie
}

Studi di Storia Contemporanea

$N^{\circ} 35,3 \mid 2018$

Gli strumenti di Clio

\section{Il progetto campifascisti.it}

\section{Andrea Giuseppini}

\section{(2) OpenEdition \\ Journals}

\section{Edizione digitale}

URL: http://journals.openedition.org/diacronie/9237

DOI: 10.4000/diacronie.9237

ISSN: 2038-0925

\section{Editore}

Association culturelle Diacronie

\section{Notizia bibliografica digitale}

Andrea Giuseppini, « II progetto campifascisti.it », Diacronie [Online], № 35, 3 | 2018, documento 16, Messo online il 29 septembre 2018, consultato il 01 mai 2019. URL : http://journals.openedition.org/ diacronie/9237 ; DOl : 10.4000/diacronie.9237 


\section{Diacronie}

Studi di Storia Contemporanea

\section{$35,3 / 2018$}

Gli strumenti di Clio: uomini, luoghi e teorie della storia dalla tradizione critica alla comunicazione digitale

\section{Il progetto campifascisti.it}

\section{Andrea GIUSEPPINI}

Per citare questo articolo:

GIUSEPPINI, Andrea, «Il progetto campifascisti.it», Diacronie. Studi di Storia Contemporanea : Gli strumenti di Clio: uomini, luoghi e teorie della storia dalla tradizione critica alla comunicazione digitale, 35, 3/2018, 29/09/2018,

URL: < http://www.studistorici.com/2018/09/29/giuseppini_numero_35/ >

Diacronie Studi di Storia Contemporanea $\rightarrow$ http://www.diacronie.it

Rivista storica online. Uscita trimestrale.

redazione.diacronie@hotmail.it

Comitato di direzione: Naor Ben-Yehoyada - João Fábio Bertonha - Christopher Denis-Delacour - Maximiliano Fuentes Codera Anders Granås Kjøstvedt - John Paul Newman - Deborah Paci - Niccolò Pianciola - Spyridon Ploumidis - Wilko Graf Von Hardenberg

Comitato di redazione: Jacopo Bassi - Luca Bufarale - Gianluca Canè - Fausto Pietrancosta - Alessandro Salvador - Matteo Tomasoni - Luca Giuseppe Manenti

Diritti: gli articoli di Diacronie. Studi di Storia Contemporanea sono pubblicati sotto licenza Creative Commons 3.0. Possono essere riprodotti e modificati a patto di indicare eventuali modifiche dei contenuti, di riconoscere la paternità dell'opera e di condividerla allo stesso modo. La citazione di estratti è comunque sempre autorizzata, nei limiti previsti dalla legge. 


\title{
16/ Il progetto campifascisti.it
}

\author{
Andrea GIUSEPPINI
}

L'obiettivo del contributo è presentare brevemente il sito www.campifascisti.it, illustrare i suoi intenti e rendere conto dei principali modi in cui è stato di fatto utilizzato dal pubblico. La valutazione è nel complesso positiva.

\section{Introduzione}

Online dal 2012, il progetto campifascisti nasce in ambito non accademico grazie ad un finanziamento di 56.000,00 euro ottenuto partecipando al bando Europe for Citizens - European Remembrance $^{1}$. In origine, gli obiettivi principali del progetto erano due: raccogliere quante più testimonianze orali di ex internati fosse possibile compatibilmente con le risorse economiche e di tempo disponibili, e realizzare una mappatura on line dei campi di concentramento istituiti durante tutto il corso della dittatura fascista: dai primi campi realizzati in Libia nel 1930 su ordine del generale Rodolfo Graziani, fino a quelli creati dopo l'8 settembre 1943 dalla Repubblica di Salò. Rispetto a questo secondo obiettivo, il progetto si ancorava saldamente alla produzione storiografica sul tema che a partire dalla seconda metà degli anni Ottanta si era andata via via arricchendo di studi a carattere generale, relativi a particolari territori, o su singoli campi di concentramento $^{2}$. Una mappatura, quella rintracciabile nella produzione scientifica, che si può dire fosse già allora pressoché completa per quanto riguarda il territorio italiano e quelli annessi

\footnotetext{
${ }^{1}$ Il progetto è stato ideato da Andrea Giuseppini e Roman Herzog per l'associazione Audiodoc. Dal 2015 il progetto è curato dall'associazione Topografia per storia.

${ }^{2}$ Per una cronologia degli studi sull'internamento e un'esaustiva rassegna bibliografica si veda CAPOGRECO, Carlo Spartaco, «Tra storiografia e coscienza civile. La memoria dei campi fascisti e i vent'anni che la sottrassero all'oblio», in Mondo contemporaneo, 2/2014, pp. 137-166.
} 
o occupati in Jugoslavia nel corso del secondo conflitto mondiale, e lacunosa soltanto per l'Africa, la Grecia, l'Albania e la Francia.

L'idea era dunque quella di cercare di colmare queste lacune e quindi di raccogliere in un unico strumento on line quante più informazioni e dati possibili su tutti i campi, "vecchi" e "nuovi", attingendo a tre fonti principali: la letteratura storiografica disponibile, le fonti archivistiche e le testimonianze. Questo strumento avrebbe inoltre offerto agli utenti una visione d'insieme anche in senso temporale, ricucendo su un'unica linea del tempo i diversi periodi analizzati dagli studi settoriali.

Tuttavia, fin dall'inizio del progetto ci si è resi conto che limitare la mappatura ai soli campi di concentramento avrebbe offerto una visione parziale - seppur rilevante - degli strumenti e delle pratiche di reclusione adottate dal fascismo nei confronti di singoli individui o di gruppi più o meno ampi di popolazione. Affiancare alla mappatura dei campi di concentramento anche le località di confino, i luoghi di internamento cosiddetto libero, le carceri e così via, avrebbe a nostro avviso fornito un quadro più ricco delle diverse pratiche di reclusione considerandole come componenti di un sistema unitario, e permesso di comprendere e valutare meglio la funzione stessa dei campi di concentramento fascisti offrendo termini di confronto in fatto di genesi, livelli di violenza, tipologie di vittime, ecc.

È chiaro che porsi un simile obiettivo significa condannarsi quasi inevitabilmente all'incompletezza. Tuttavia, questa incompletezza è un grave limite nel caso in cui si voglia produrre un saggio, un rapporto o un libro, ma non dove si sia scelto come strumento di lavoro e pubblicazione un sito web, ossia un prodotto che è possibile modificare, arricchire, rivedere, ecc., nel tempo, in funzione delle scoperte e verifiche fatte.

Per esempio, di recente, abbiamo incluso nella mappatura una nuova tipologia di luogo, ossia gli "Istituti di rieducazione per minorenni". Per il momento ne abbiamo individuati sei $^{3}$. Vi vennero reclusi dal settembre 1942 ventitré minorenni jugoslavi condannati dal Tribunale speciale per la Dalmazia per il reato di associazione sovversiva. Si tratta di luoghi che avrebbero dovuto "rieducare" più che punire e che dunque ospitavano i ragazzi fino alla maggiore età «o fino a quando avessero dato prove evidenti di ravvedimento». Lo chiarisce rispondendo a una lettera di un genitore il direttore dell'istituto di Bosco Marengo: «vostro figlio è ricoverato in questa Casa di Rieducazione, non detenuto, non essendo questo un Istituto per detenuti, ma per giovanetti che abbiano bisogno di essere riavviati alla vita sociale». Una forma di internamento

\footnotetext{
${ }^{3}$ Gli istituti di rieducazione minorile cui ci riferiamo si trovavano a Bosco Marengo (Alessandria), Parma, Urbino, Verbania, Torino e Roma (in quest'ultimo caso si tratta di una minorenne assegnata all'istituto femminile del Buon Pastore).
} 
che ricorda, almeno nella sua funzione "rieducativa", i campi di concentramento per minorenni", maschi e femmine, sottratti alle famiglie di "ribelli" dopo la riconquista della Libia.

O ancora: accumulando documenti sulle carceri, siamo riusciti a vedere che nel corso della seconda guerra mondiale questi istituti hanno avuto la funzione di luoghi di reclusione anche per civili provenienti dai paesi occupati, in particolare Jugoslavia e Grecia, condannati dai Tribunali militari di guerra, ossia di detenuti politici processati nei loro paesi per "atti contro l'esercito italiano" da un'istituzione militare ma deportati in Italia e reclusi in istituti gestiti dal Ministero di Grazia e giustizia. Non è ancora noto il numero di questi detenuti. Grazie al ritrovamento di una rubrica inedita tutt'oggi conservata presso l'Istituto di pena di Parma, sappiamo che dal luglio del 1942 ai primi mesi del 1944 furono reclusi in questo carcere almeno 865 civili, tutti di nazionalità jugoslava, tranne un piccolo numero di greci. Sulla base di altri documenti già pubblicati sul nostro sito, possiamo ipotizzare che le nostre carceri abbiano ospitato in quegli anni circa 2-3.000 stranieri condannati dai Tribunali militari di guerra. Vale la pena di osservare che il ruolo giocato da questa istituzione nella repressione dei "ribelli" è solo da alcuni anni oggetto di attenzione da parte degli storici, che infatti hanno fin qui privilegiato lo studio di un altro strumento di repressione utilizzato dal Regio esercito italiano, ossia l'internamento in campi di concertamento e in località, una forma di internamento indiscriminata che non prevedeva alcun processo.

Estendendo il progetto di mappatura anche alle località di internamento ci siamo posti due obiettivi. In primo luogo provare ad arrivare a un elenco completo di tutti i comuni sede dell'internamento cosiddetto libero. Se infatti si conoscono probabilmente tutte le località di internamento per ebrei stranieri ${ }^{5}$, e sono noti, grazie a diversi studi, molti dei luoghi di internamento di alcune specifiche regioni e province, il quadro resta incompleto non soltanto per quando riguarda la mappatura dei luoghi, ma anche perché poco si sa della storia dei luoghi noti (date di funzionamento, numero e nazionalità degli internati, condizioni di vita, ecc.).

Il secondo obiettivo è cercare di ricostruire in maniera lineare, senza salti temporali e di luogo, i percorsi di prigionia. Ad esempio, tenendo conto dei luoghi di internamento siamo riusciti a tracciare con precisione la storia di reclusione di circa 250 civili deportati in Italia dopo la strage di Podhum, una località oggi in Croazia dove nel luglio del 1942 furono fucilati per rappresaglia quasi cento civili e incendiate tutte le abitazioni, e dalla quale fu deportata l'intera popolazione. Molti di essi furono inizialmente assegnati al campo di concentramento di Lovran, quindi divisi in piccoli gruppi familiari composti prevalentemente da donne, bambini e anziani (essendo i maschi adulti morti fucilati) e internati in numerosi piccoli comuni del nord Italia: da Spotorno in

\footnotetext{
${ }^{4}$ Facevano parte di questo sistema i campi di concentramento per minorenni presso le località di Agedabia, Marsa al Brega, Sidi Ahmed el-Magrun, Soluch e Zliten.

${ }^{5}$ Anna Pizzuti ha compilato, oltre a un documentato data base con i nomi degli ebrei stranieri internati in Italia, anche una lista delle località di internamento. Cfr. URL: < http://www.annapizzuti.it > [consultato il 18 settembre 2018].
} 
provincia di Savona, a Sant'Agostino in provincia di Ferrara, da Ponte in Valtellina (Sondrio) a Invorio, Divignano e Carpignano Sesia (Novara), e così via. Deportati con i soli abiti estivi (quelli indossati al momento della cattura), furono costretti a vivere miseramente, alloggiati in locali privi di tutto o presso ricoveri per poveri. Finché, nel gennaio del 1943, furono infine trasferiti presso il nuovo campo di concertamento Le Fraschette di Alatri in provincia di Frosinone.

Un'altra parte del progetto ancora da sviluppare è quella riguardante le località di confino. Vale la pena di sottolineare che nella nostra ricerca non ci si occuperà comunque soltanto del confino politico, ma anche, almeno in una certa misura, del confino comune. Grazie ad alcune ricerche condotte negli archivi della Slovenia, abbiamo infatti scoperto che in preparazione dell'entrata in guerra il fascismo ha fatto ricorso al confino comune per mettere sotto controllo l'intera comunità sinti e rom che viveva in Istria, assegnandola ad alcune località della Sardegna. Ebbero lo stesso destino anche alcuni sinti italiani residenti in Basilicata.

Infine si è rivelata di qualche utilità anche l'inclusione nella mappatura dei campi per prigionieri di guerra, nonostante si tratti ovviamente di campi con una funzione molto diversa da quella dei campi per internati civili. Non solo infatti siamo riusciti a compilare un elenco pressoché completo dei campi per prigionieri di guerra gestiti dal Regio esercito italiano, ma la documentazione raccolta ci ha anche consentito di mettere in risalto le differenze di trattamento riservate ai prigionieri di guerra jugoslavi ${ }^{6}$ rispetto a quelli appartenenti agli eserciti alleati. È il caso del sistema di campi per prigionieri jugoslavi in funzione in Sardegna dal settembre del 1941 al luglio 1943. Ci riferiamo al campo di Carbonia e ai suoi distaccamenti, in cui, senza alcun controllo e tutela da parte degli organismi internazionali, furono costretti al lavoro forzato nelle miniere circa 2.200 prigionieri (1.800 jugoslavi e 400 greci). Secondo i documenti di fonte jugoslava, almeno 70 di loro morirono a causa delle pesanti condizioni di lavoro, delle pessime condizioni igieniche e della fame. Non solo dunque furono trattati in modi simili a quelli riservati ai civili nei campi per internati civili gestiti dal Regio esercito italiano; toccò loro anche il lavoro forzato.

Ad oggi sul sito sono mappati 1.039 luoghi divisi per tipologia ${ }^{7}$. Per molti di essi è stata redatta una scheda sintetica. Ogni luogo è accompagnato da una serie di informazioni (documentazione, testimonianze, immagini e bibliografia). La mappatura consente di generale elenchi per paese,

\footnotetext{
${ }^{6}$ I vertici dell'esercito italiano decisero che i militari jugoslavi catturati nel corso della campagna militare di occupazione del paese (aprile 1941) e mai rilasciati dovessero essere considerati come prigionieri di guerra. I militari catturati e rilasciati al termine delle operazioni belliche se ricatturati nel corso dei mesi successivi saranno internati come civili.

${ }^{7}$ Riguardo alla tipologia dei luoghi si è deciso di seguire la definizione che compare con più frequenza nella documentazione primaria. Quindi un luogo indicato prevalentemente come campo di concentramento (e non di transito, di lavoro, di internamento, ecc.) è classificato nella nostra tipologia come, appunto, "campo di concentramento".
} 
regione, provincia, tipologia, funzione, nazionalità degli internati e dipendenza. Il data base dei documenti contiene oltre 3.300 copie digitali provenienti da numerosi archivi, non solo italiani ${ }^{8}$.

\section{Uso pubblico del sito campifascisti.it}

A sei anni dalla pubblicazione online del progetto può essere di qualche interesse fornire alcuni dati sulla fruizione del sito da parte del pubblico. Dal 2012 il sito ha avuto in media 60 visite al giorno, una media che si è mantenuta costante nel corso degli anni. Essa tiene conto di un picco di visite che si verifica ogni anno in occasione del "Giorno della memoria", il 27 gennaio, quando le consultazioni salgono a circa 600-700. La durata media di ogni visita è di 4,30 minuti, nel corso dei quali vengono visualizzate in media 4,5 pagine. Le pagine più visitate sono quelle relative alle schede dei luoghi e ai documenti. È un buon risultato ma ovviamente aspiriamo a un risultato migliore. Né è difficile spiegare perché il numero delle visite sia ancora così contenuto: il tema probabilmente non è tra quelli di maggiore richiamo sul web; il sito è solo in lingua italiana benché potenzialmente interessante anche per visitatori di altri paesi, primi fra tutti quelli di provenienza degli internati; il progetto è gestito da una piccola associazione non sostenuta da alcun ente di ricerca, istituzione accademica, istituto storico o fondazione e priva di risorse da investire nella comunicazione.

Appassionante e gratificante è stato invece il rapporto in termini di progetto partecipato con quanti hanno dimostrato interesse alla nostra ricerca. Coinvolgere altri era ed è in effetti uno dei principali intenti del progetto. I rapporti intrecciati con il pubblico e con diverse istituzioni stimolati dal sito sono stati e sono tutt'ora di diverso tipo. Grazie all'apporto di singoli individui il nostro data base si è arricchito, ad esempio, delle riproduzioni di una serie di cartoline inviate dagli internati dai relativi campi di concentramento, raccolte da Veselko Gustin, figlio di un ex internato sloveno; di alcune immagini inedite provenienti da archivi privati di famiglia (due foto del campo di concentramento di Kavaja in Albania e una serie di immagini relative alla costruzione delle baracche del campo per prigionieri di guerra presso la miniera di Monteponi in Sardegna); di foto di scritte incise sui muri dai prigionieri di guerra greci detenuti nel convento di San Romano a Montopoli in Val d'Arno; di mappe di campi e di altre foto del periodo. L'archivio di stato di Ancona ci ha proposto di pubblicare on line alcuni documenti da esso conservati sui campi di concentramento di Sassoferrato e Fabriano. Il Dipartimento di lingue e letterature dell'Università di Udine ha utilizzato alcune delle testimonianze orali di ex internati croati da noi raccolte, nell'ambito di un tirocinio di traduzione verso l'italiano e come materiale di una tesi di

\footnotetext{
${ }^{8}$ Ricordiamo i più importanti: Archivio centrale dello stato, Archivio storico del ministero degli esteri, Archivio storico dello Stato maggiore dell'esercito, Archivio storico della Croce Rossa Italiana, Archivio di Stato della Slovenia, International Tracing Service di Bad Arolsen.
} 
laurea magistrale in traduzione di storia orale, e in cambio ci fornirà le traduzioni da noi mai realizzate per mancanza di finanziamenti. Queste stesse testimonianze orali sono oggetto anche di uno scambio con l'Istituto di etnologia e folclore di Zagabria che le ha trascritte e le conserverà nel proprio archivio nell'ambito del progetto Narrating fear e che dal canto suo ci fornirà altre interviste a ex internati del campo di concentramento di Molat da loro da poco realizzate.

Particolarmente apprezzato e condiviso in quanto fonte di documentazione primaria è il nostro data base dei documenti. Ad esempio, diversi autori del recentissimo terzo volume dell'Encyclopedia of Camps and Ghettos curata dall'United States Holocaust Memorial Museum di Washington dedicato ai campi di concentramento istituiti nei o dai paesi europei alleati dei nazisti, citano i documenti pubblicati sul nostro sito. Sono basate sul nostro lavoro, ad esempio, le schede relative alle località di internamento per ebrei presenti in Dalmazia. Relativamente ai campi italiani in Africa orientale, l'Encyclopedia prende atto della carenza dei documenti disponibili e, rinunciando a stendere schede relative ai singoli luoghi, pubblica esclusivamente la mappatura compilata da campifascisti. Citano documenti contenuti nel data base anche diverse voci di wikipedia e alcuni saggi storici. Numerose sono state anche le richieste di utilizzare i documenti da noi pubblicati in piccole mostre e percorsi didattici. Benché un data base contenente una selezione di documenti non possa in alcun modo sostituire una ricerca archivistica, accedere molto facilmente a un largo insieme di fonti primarie conservate in archivi diversi e non sempre facilmente accessibili, si dimostra un'opportunità molto apprezzata anche per chi intende procedere a una vera ricerca storica.

Gran parte del nostro data base dei documenti e dei luoghi, in tutto 3.683 record, è confluito nel portale del Ministero dei beni culturali www.culturaitalia.it, passo necessario per poi entrare a far parte del progetto archivistico digitale dell'Unione europea www.europeana.eu.

Una delle aspirazioni del progetto "campifascisti" è sensibilizzare il pubblico e le istituzioni sul valore di ciò che rimane dei campi di concentramento in quanto luoghi della memoria e promuoverne la conservazione. Per esempio, grazie alla nostra scheda, l'assessore alla cultura del comune di Le Thuile in Valle d'Aosta, ha "riscoperto" la storia del campo per prigionieri di guerra jugoslavi di Porta Littoria, il nome assegnato al paese durante il fascismo. I circa 200 prigionieri jugoslavi reclusi in questo luogo erano costretti al lavoro presso una miniera di carbone. Facendo leva sui documenti da noi pubblicati, l'assessore ha cercato di contrastare un progetto di abbattimento delle baracche ancora esistenti per fare spazio a una serie di capannoni destinati ad attività artigianali, ottenendo in un primo momento l'impegno del comune a salvaguardare una delle baracche da destinare a museo del campo. Sembra tuttavia che la giunta comunale abbia di recente rimesso mano al progetto stabilendo l'abbattimento di tutte le baracche, tanto che l'associazione Legambiente della Valle d'Aosta ha deciso di assegnare per questo la "bandiera nera" all'amministrazione comunale. 
Infine, la consultazione dei molti materiali - documenti, schede, immagini e testimonianze pubblicati da campifascisti sull'internamento in Italia della comunità maltese residente in Libia ha suggerito a un giornalista della televisione di stato maltese di realizzare un documentario in due puntate sull'argomento. È una storia poco nota anche a Malta, ma di notevole interesse in particolare perché si inserisce nel più ampio progetto fascista di dominio del Mediterraneo e di rivendicazione della italianità di Malta. Grazie ai nostri contatti, l'autore ha potuto intervistare alcuni degli ultimi testimoni diretti della deportazione maltese, oggi residenti in Australia, e ha poi visitato in nostra compagnia i campi di concentramento di Fraschette di Alatri e di Fossoli, ossia delle due principali tappe dell'internamento dei 1.700 circa maltesi tripolini portati in Italia. Il documentario andrà in onda il prossimo autunno.

Sarà banale dirlo ma solo lo strumento del web è in grado di dare legittimità a un lavoro in progress e consentire la realizzazione di progetto partecipato. 


\section{L'AUTORE}

Andrea GIUSEPPINI è curatore del progetto campifascisti, uno dei soci fondatori dell'associazione Topografia per la storia, e autore di diversi audio documentari di ricostruzione storica.

URL: < http://www.studistorici.com/progett/autori/\#Giuseppini > 